\title{
Complexity-Reduced Multilevel Coding with Rate-Compatible Punctured Convolutional Codes
}

\author{
Jung-Fu Cheng, Ching-Hua Chuang, and Lin-shan Lee \\ Department of Electrical Engineering, Rm. 512 \\ National Taiwan University \\ Taipei, Taiwan, Republic of China
}

\begin{abstract}
A new coding design with large coding gains and complexity-reduced decoders is proposed. The coding strategy is based on a multilevel coding construction with rate-compatible punctured convolutional codes as building blocks. A new sub-optimal decoding is proposed where only one decoder using Viterbi algorithm is drafted. Typical example codes for quadrature amplitude shift keying (QASK) constellations are constructed and shown to outperform Ungerboeck's codes, but with higher decoding delay. Computer simulation is also performed to verify the results.
\end{abstract}

\section{Introduction}

Since Ungerboeck proposed the concept of mapping by set partitioning to achieve significant coding gains without sacrificing bandwidth [1], substantial research efforts have been made in the design of bandwidth-efficient codes to achieve even larger gains [29]. However, a main obstacle in the practical application of such power- and bandwidth-efficient coded systems lies in the complexity of decoders at the receiver end. For example, as an essential component for various coded modems, Viterbi decoder requires bulky hardware which grows with the number of states, especially for highspeed applications and high rate codes. This has limited the number of states used in most trellis coded modulation (TCM) applications (typically up to 16 and rarely more [2]), because rate $2 / 3$ codes are used. On the other hand, Viterbi decoders of speed up to $25 \mathrm{MHz}$ with 64 states and rate 1/2 are now commercially available, which can be punctured to various higher rates such as up to $15 / 16$. This leads to the proposal and recent commercial products of pragmatic TCM by Viterbi et. al. [2], where rate 1/2 decoders are used while the coding gains are maintained.

In this paper, a new coding scheme based on multilevel coding structures [3-9] is proposed, which achieves larger coding gains while requiring only one Viterbi decoder of rate $1 / 2$ or $1 / 3$. This is made

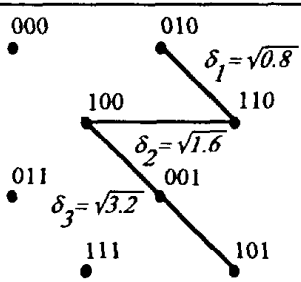

$$
\underbrace{0000}_{\delta_{4}=\sqrt{3.2}}
$$

Fig. 1. Signal constellations and labels of 8AMPM and 16QASK. possible by employing rate-compatible punctured convolutional (RCPC) codes [10] and the introduction of interblock memory designs in multilevel codes $[6,7]$. In the following, we will begin by first briefly reviewing these previous results in section 2 . Then, the new coding scheme and several examples are presented in section 3. Section 4 introduces interleaving and iterative decoding techniques to further improve the new coding strategy and numerical simulation results are provided for verification. Section 5 finally makes our concluding remarks.

\section{Brief Review of Some Preliminaries}

\subsection{Multilevel Coding with Interblock Memory}

In a multilevel coding system, a redundant constellation is binarily partitioned $\boldsymbol{m}$ times into subsets with minimum intra-set distances $\delta$, $i=1$ to $m$, that are nondecreasing with $i$. Each symbol is then labeled by the partition chain [5] (some typical examples are shown in Fig. 1). Each of the $m$-bits defining one of the $M=2^{m}$ symbols is associated with a code that will be encoded and decoded by different codec. For example, the block diagrams of a typical encoder and decoder for conventional multilevel codes with $m=3$ are shown in Fig. 2 (for convenience of discussion, we will first concentrate on this case in the following and then generalize the principle to other constellations in later examples). In the encoding process, the encoders of the three levels receive demultiplexed input bits $\mathbf{a}, \mathbf{b}, \mathbf{c}$ and then encode them independently into $x, y, z$ The mapper accepts the coded bits $x_{j} y_{j} z_{j}$ to select a symbol for transmission. The

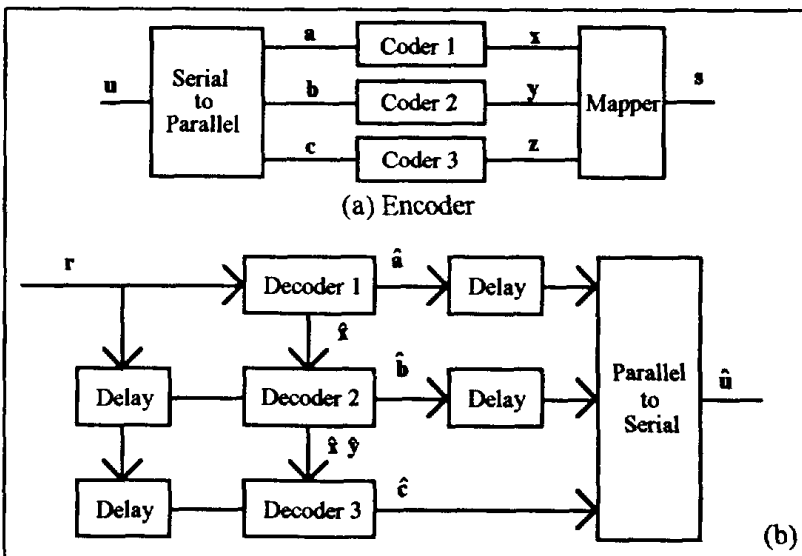

Decoder

(b)

Fig. 2. Block diagrams of conventional multilevel cadec. 


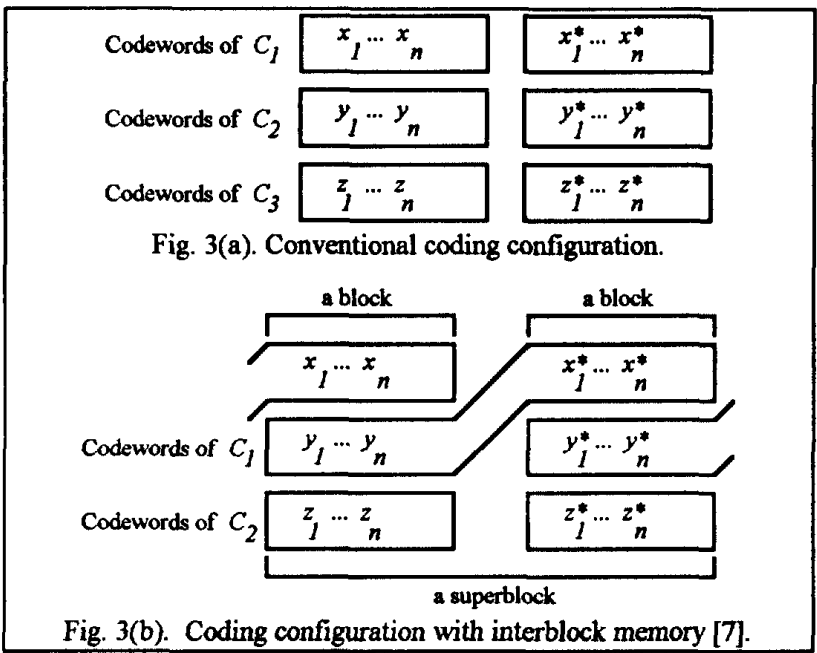

decoding process is performed by a multi-stage algorithm for reasons of complexity. The received signals $\mathbf{r}$ are first fed to the decoder of level 1 to estimate the transmitted level 1 information bits $\mathbf{~}$. These bits are reencoded as $\mathbf{I}$ and then delivered to level 2 decoder, which extracts the level 2 information bits based on the information from level 1 decoder. Finally, level 3 information bits are determined with the information provided by levels 1 and 2 decoders.

In general, various codes used in each of the coding levels require different encoders and decoders. Furthermore, if higher gains are desired, "stronger codes" (longer block codes or convolutional codes with larger constraint length) are usually applied. The resulting decoders might easily become formidably complex and unpractical (if not impossible) to implement. A new design concept called interblock memory has been proposed recently $[6,7]$, which might be a promising approach to circumvent these constraints. In this scheme, unlike conventional systems where the three levels of codes are applied independently as shown in Fig. 3(a), the coding designs for every two adjacent blocks are related. As shown in Fig. 3(b), code $C$, has a code length of twice the block length so that the coded modulation of each block depends on the preceding block. The decoding algorithm therefore regards two adjacent code blocks as a superblock. For each superblock, by assuming $\left(x_{1}, \ldots, x_{n}\right)$ is correct, $\left(y_{1}, \ldots, y_{n^{*}} x_{1}^{*}, \ldots, x_{n}{ }^{*}\right)$ is decoded first and then reencoded to obtain the estimates $\left(\hat{y}_{1}, \ldots, \hat{y}_{n} \hat{x}_{1}^{*}, \ldots, \hat{x}_{n}^{*}\right)$. Consequently, $\left(z_{1}, \ldots, z_{n}\right)$ can be decoded next. The decoding of $\left(y_{1}^{*}, \ldots, y_{n}^{*}\right)$ and $\left(z_{1}^{*}, \ldots, z_{n}^{*}\right)$ could be proceeded similarly in the next superblock since the estimates of $\left(x_{1}^{*}, \ldots, x_{n}^{*}\right)$ is now available. This interblock coding concept offers additional design flexibility and makes higher coding gains achievable using low complexity decoding mechanisms. In the above example, since $C_{l}$ is actually formed by two shorter codes, the decoder might be easier to implement [7].

\subsection{A Special Characteristic of RCPC Codes}

A punctured code is a high rate code obtained by periodically deleting (i. e. puncturing) certain coded bits (i. e. with some rule) from the output stream of a low rate encoder. When compared with

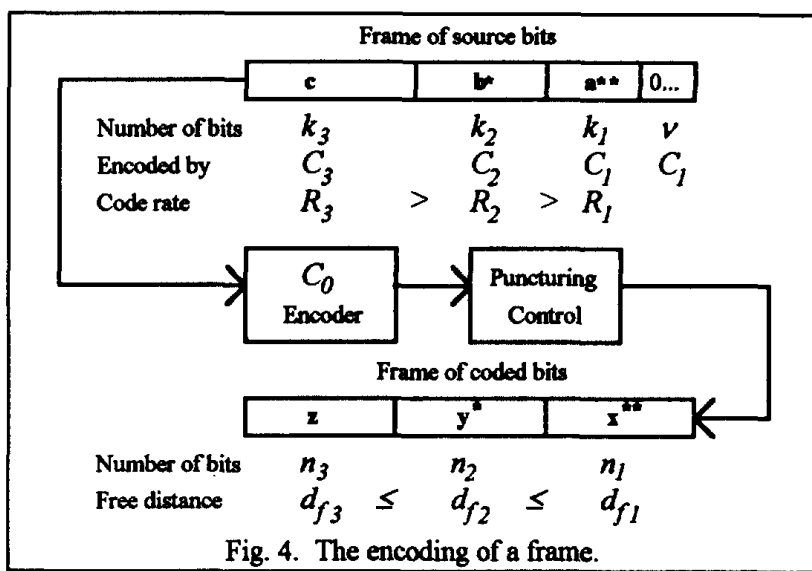

optimum codes of equal rates, punctured codes are slightly less efficient but the decoding complexity is greatly reduced. A family of RCPC codes are generated by adding a rate-compatibility restriction to the puncturing rule. This restriction implies all the coded bits of a high rate punctured code are also used by lower rate codes of the family such that a number of applications are possible [10].

Suppose we have three RCPC codes $C_{1}, C_{2}$ and $C_{3}$ of code rates $R_{1}, R_{2}$ and $R_{3}$, all derived from the same mother code $C_{0}$ of constraint length $v$ by three puncturing rules $P_{1}, P_{2}$ and $P_{3}$. respectively. In general, if $R_{1}<R_{2}<R_{3}$, we have their free distances $d_{f_{1}} \geq d_{f_{2}} \geq d_{f_{3}}$. Suppose there are three sections of information bits $\mathrm{c}, \mathrm{b}^{\star}, \mathrm{a}^{\star \star}$, each of $k_{3}, k_{2}$ and $k_{1}$ bits, respectively, chained one following another and then appended by 0 's of length $v$ as shown in Fig. 4. These chained sections are viewed as a frame of source bits and fed to a $C_{0}$ encoder serially. During bits of $\mathrm{c}$, the puncturing rule $P_{3}$ is used by the puncturing control. As soon as the first bit of $b^{*}$ enters the encoder, the puncturing rule is switched to $P_{2}$ and will be used until the end of $b^{*}$. For bits of $a^{n *}$ and the appended 0 's, the puncturing rule is $P_{l}$. Of course, after the $v 0^{\prime} \mathrm{s}$, the encoder is forced back to the zero state. The three sections of $\mathbf{c}, \mathbf{b}^{*}$, and $a^{* *}$ with appended 0 's are encoded into three sections of coded bits $\mathrm{z}, \mathrm{y}^{\star}$ and $\mathrm{x}^{* *}$ of length $n_{3}, n_{2}$ and $n_{1}$, respectively. The aggregate of $z, y^{*}$ and $x^{* *}$ is then viewed as a frame of coded bits. It was pointed out in [10] that this frame of coded bits, with the help of adequate puncturing control, could be decoded by only one Viterbi decoder sequentially starting from $\mathrm{z}$ to $\mathrm{y}^{*}$ and then $\mathrm{I}^{\mathrm{n} *}$, without loss of designed performance.

\section{The New Coding Scheme}

A new multilevel coding configuration using RCPC codes is proposed by integrating the interblock memory design as shown in Fig. 5 . The interconnections among blocks drawn in Fig. 5 just indicate that $\left(z_{l}\right.$, $\left.\ldots, z_{n} y_{1}^{*}, \ldots, y_{n}^{*}, x_{1}^{* *}, \ldots, x_{n}^{* *}\right)$ is a frame of coded bits constructed as in Fig. 4, with the understanding that $k_{1}, k_{2}, k_{3}$ can be carefully chosen such that $n_{1}=n_{2}=n_{3}=n$ (though this is not a strict constraint). In the encoding process, input source bits are periodically inserted with 0 's and then encoded by the above described method into frames of coded bits. Several frames of coded bits are interlaced to form 
Table 1. Coded 8AMPM

\begin{tabular}{|c|cc|c|c|c|c|c|}
\hline \multirow{2}{*}{$\begin{array}{c}\text { Number of } \\
\text { states 2 }\end{array}$} & \multicolumn{2}{|c|}{$D_{\min }{ }^{2} / \delta_{l}{ }^{2}$} & \multicolumn{2}{c|}{ ACG over uncoded QPSK (dB) } & \multicolumn{2}{|c|}{ Average Rate } \\
\cline { 2 - 8 } & $\mathrm{CC} 1$ & $\mathrm{CC} 2$ & $\mathrm{CC} 1$ & $\mathrm{CC} 2$ & $\mathrm{TCM}[1]$ & $\mathrm{CC} 1$ & $\mathrm{CC} 2$ \\
\hline 8 & 6.00 & 8.00 & 3.83 & 4.76 & 3.00 & 2.014 & 1.868 \\
16 & 7.00 & 8.00 & 4.47 & 4.74 & 3.80 & 2.000 & 1.861 \\
32 & 8.00 & 12.00 & 5.02 & 6.48 & 3.80 & 1.986 & 1.854 \\
64 & 10.00 & 12.00 & 5.96 & 6.47 & 4.50 & 1.972 & 1.847 \\
\hline
\end{tabular}

Table 2. Coded 16QASK

\begin{tabular}{|c|c|c|c|c|c|c|c|c|c|c|}
\hline \multirow{2}{*}{$\begin{array}{c}\text { Number of } \\
\text { states } 2^{\nu}\end{array}$} & \multicolumn{4}{|c|}{$D_{\min }{ }^{2} / \delta_{1}{ }^{2}$} & \multicolumn{2}{|c|}{ ACG over uncoded 8AMPM (dB) } & \multicolumn{4}{c|}{ Average Rate } \\
\cline { 2 - 12 } & $\mathrm{CC3}$ & $\mathrm{CC} 4$ & $\mathrm{CC5}$ & $\mathrm{CC} 3$ & $\mathrm{CC} 4$ & $\mathrm{CC} 5$ & $\mathrm{TCM}[1]$ & $\mathrm{CC3}$ & $\mathrm{CC} 4$ & $\mathrm{CC5}$ \\
\hline 8 & 6.00 & 8.00 & 6.00 & 4.79 & 5.83 & 4.63 & 3.97 & 3.014 & 2.868 & 2.903 \\
16 & 7.00 & 8.00 & 7.00 & 5.44 & 5.81 & 5.28 & 4.77 & 3.000 & 2.861 & 2.889 \\
32 & 8.00 & 8.00 & 8.00 & 6.00 & 5.80 & 5.84 & 4.77 & 2.986 & 2.854 & 2.875 \\
64 & 8.00 & 8.00 & 10.00 & 5.98 & 5.79 & 6.78 & 5.44 & 2.972 & 2.847 & 2.861 \\
\hline
\end{tabular}

blocks for transmission. In other words, though $\mathbf{x}^{*}, \mathbf{y}^{*}$ and $\mathbf{z}^{*}$ belong to different coded frames, the mapper uses $x_{j}{ }^{*}, y_{j}{ }^{*}, z_{j}^{*}$ to select a channel symbol, for $j=1$ to $n$. The complexity of encoder has been reduced since only one shift register based convolutional encoder and some simple puncturing control are called for, though additional buffers are required. In the decoding process, three adjacent blocks are viewed as a superblock. By virtue of the special characteristic of RCPC codes discussed in section 2.2, the frame $\left(z_{1}, \ldots, z_{n}, y_{1}^{*}, \ldots\right.$, $\left.y_{n}^{*}, x_{l}^{* *}, \ldots, x_{n}^{* *}\right)$ could be decoded by only one decoder using Viterbi algorithm, assuming that $\left(x_{l}, \ldots, x_{n}\right)$ and $\left(y_{l}, \ldots, y_{n}, x_{l}^{*}, \ldots\right.$, $\left.x_{n}{ }^{*}\right)$ are correct. The decoded source bits are reencoded to obtain the estimates $\left(\hat{y}_{1}^{*}, \ldots, \hat{y}_{n}^{*}, \hat{x}_{1}^{* *}, \ldots, \hat{x}_{n}^{* *}\right)$, which will be used in the decoding of the next superblock. The decoding of $\left(z_{1}^{*}, \ldots, z_{n}^{*}, y_{l}^{* *}\right.$, $\left.\ldots, y_{n}^{* *}, \ldots\right)$ is proceeded similarly within the next superblock. A major advantage of this coding scheme becomes obvious now: the complexity of decoding mechanism is significantly reduced because only one Viterbi decoder is required to decode all the three levels of coded bits

For a simplified evaluation of the performance, asymptotic coding gains (ACG) will be considered. The minimum squared Euclidean distance $D_{\min }{ }^{2}$ between reemerging code sequences is at least $\min \left(\delta_{1}{ }^{2} d_{f_{1}}, \delta_{2}{ }^{2} d_{f_{2}}, \delta_{3}{ }^{2} d_{f_{3}}\right)$ [3-5]. ACG is then defined as $\left(D_{\min } D_{\text {ref }}\right)^{2}$, where $D_{\text {ref }}$ is the minimum Euclidean distance for the uncoded reference scheme computed on the basis of the same $E_{b} / N_{0}$. To compare complexity of the new coding scheme with others, the average number of branches per decoded bit in a Viterbi algorithm to

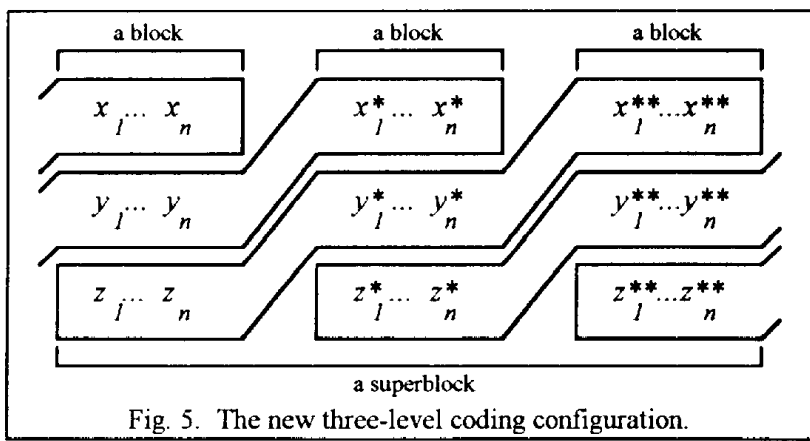

be evaluated is calculated. (The average complexity of RCPC codes is $2 * 2^{v}$ [10], whereas a general $K / N$ convolutional code of constraint length $v$ requires $2^{K} 2 v / K$ such operations.) Bandwidth efficiency is another important performance factor that will be termed in the average rate, which is defined as average information bits that can be carried by a channel symbol. We next shall examine the performance of the proposed new coding strategy in several examples in the following with TCM [1] as the baseline for comparison.

Example 1: We first construct a coded 8AMPM by coding configuration $\mathrm{CCl}$ with the three $\mathrm{RCPC} \operatorname{codes} C_{1}, C_{2}$ and $C_{3}$ of rates $R_{1}=1 / 2, R_{2}=2 / 3$ and $R_{3}=8 / 9$, respectively [10]. Since the block length $n$ is selected to be 72 , there are totally 216 coded bits but only (148v) information bits within a block. Therefore every symbol carries an average of (148- $v) / 72$ information bits. As listed in Table 1, the bandwidth efficiency of the new code is roughly the same as uncoded QPSK and sometimes better. However, it is noted here that this efficiency could be improved by using longer blocks, though this will introduce more decoding delay. The decoding complexity of this system is $2 * 2^{v}$, since the number is identically $2 * 2^{v}$ for each level. The complexity of TCM is also $2 * 2^{v}$, because a rate $2 / 3$ convolutional code is used. As also shown in Table 1, the new scheme achieves improvements of coding gains ranging from 0.67 to $1.52 \mathrm{~dB}$ over TCM. It is possible to design a second coding configuration $\mathrm{CC} 2$ which is structurally very similar to $\mathrm{CCl}$ by selecting the RCPC codes $C_{1}, C_{2}$ and $C_{3}$ to be of rates $R_{1}=1 / 3$, $R_{2}=2 / 3$ and $R_{3}=8 / 9$, respectively [10], and the block length $n$ to be 144. This configuration results in larger coding gains but with a little reduction in information rates.

Example 2: Now we shall generalize the coding configuration to four levels (i.e. $m=4$ ), which can be suitable for constellations such as 16QASK. Firstly, let the first three levels use the same RCPC codes as in example 1 and leave the fourth level uncoded as shown in Fig. 6 , that is, coding configurations $\mathrm{CC} 3$ and $\mathrm{CC} 4$ are constructed by selecting the codes to be of rates $R_{1}=1 / 2$ (for CC3) or $1 / 3$ (for CC4), $R_{2}=2 / 3, R_{3}=8 / 9$ and $R_{4}=1$ (uncoded), respectively. Since there are uncoded bits, the average decoding complexity is somewhat reduced as compared to that of $2 * 2^{v}$ for TCM. The performance parameters listed in Table 2 show that the new scheme outperforms TCM, to 


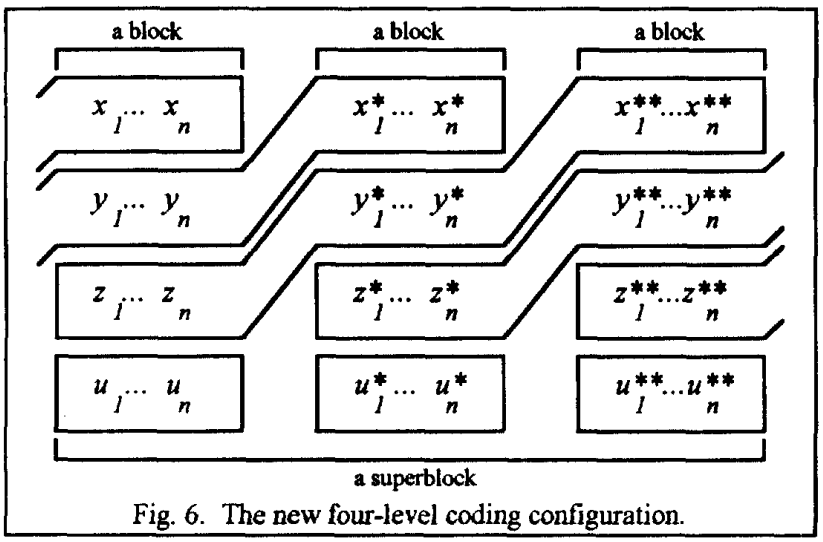

However, it also suffers from a same problem with TCM, i.e., the coding gains do not necessarily increase with the number of states, as could be found in the last two rows of Table 2 . This is because the uncoded level bounds the distance performance. Therefore, another coding configuration $\mathrm{CC} 5$ is modified from $\mathrm{CC} 3$ by encoding the fourth level with a RCPC code of rate $R_{4}=8 / 9$, too (the construction of such encoded frames is very similar to that described in section 2.2). As also listed in Table 2, this design results in a very large coding gain for the case of $v=6$, but with the loss of complexity reduction and bandwidth efficiency. Thus it would be practically unnecessary to encode the fourth level except for this case.

\section{Interleaving and Iterative Decoding}

It has been demonstrated recently that it is hard to approach the asymptotic coding gains of multilevel coding systems using all convolutional codes for bit error rates above $10^{-6}[8]$, partly because of the burstiness of errors in the reencoded sequences of Viterbi decoders output and the lack of information from lower levels when decoding upper levels. The bursty errors among reencoded input bits will make the Viterbi decoding of lower levels significantly degraded and result in an error propagation phenomenon. Without information from lower levels, the decoders of upper levels are forced to consider more possible combinations and thus increase the number of nearest neighbors of code trellis, which in turn renders a bad decoding performance. Several countermeasures are considered in [9] to overcome these adversaries, including interleaving between coded sequences of each level to remove the burstiness and iterative decoding to circumvent the later situation. The essential point of iterative decoding is to regard the decisions made after first decoding step of all levels as tentative. These decisions are then reencoded to help a second run of decoding. Since the decoder could use information about lower levels at this time, it could make more global decisions and thus improve the performance. The design of interleaver should make error bursts in the reencoded sequences of any two levels spreaded over the third. That is, the decoding of all three levels will combat with no bursty enrors in its input. We adopt here, for the example of $m=3$, a square interleaver of $L$ rows and $W$ columns with the product $L W$ equal to the block length $n$. Each cell of the interleaver contains room for three bits. Coded bits are written into the interleaver with the following rules. Coded bits of the first

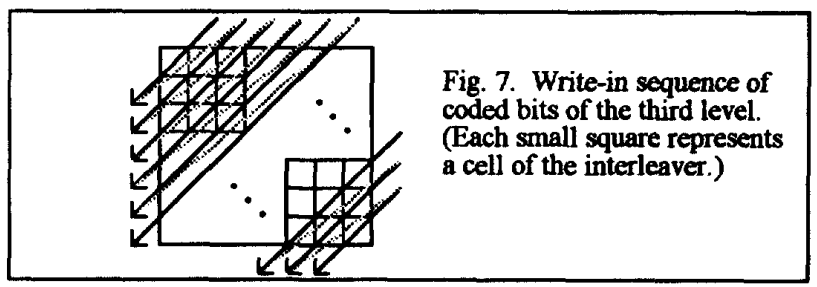

level are written row-wise into the interleaver, and column-wise for those of the second level. Coded bits of the third level are written into the interleaver in the way shown in Fig. 7. This interleaver could actually be applied in $\mathrm{CCl}$ through $\mathrm{CC} 4$, since the uncoded bits of fourth levels in $\mathrm{CC} 34$ do not need interleaving.

Computer simulation results performed for the coding configurations 1 through 4 with 8 states under an additive white Gaussian noise channel are shown in Fig. 8 and 9. Both the simulation of original designs and systems equipped with interleaving of size $L W=24 \times 24$ and iterative decoding of two iterations are performed. The introduction of these two techniques brings an addition of $0.5-0.7 \mathrm{~dB}$ coding gains to the systems. The resulting coded 8AMPM and 16QASK achieve coding gains as high as 3.8 and $4.6 \mathrm{~dB}$ over uncoded systems, respectively.

\section{Conclusions}

A new multilevel coding design with rate-compatible punctured convolutional codes is proposed using the concept of interblock memory. Several coded QASK systems are constructed and shown to achieve very high coding gains and requires relatively simple decoders. These schemes have a rather uniform structure and could be constructed by standard at hand building blocks. All these improvements call the price of longer decoding delay. The bandwidth efficiencies of these new coding systems are roughly the same as their reference systems, though can be improved by using longer block length. We have also tried interleaving and iterative decoding to improve the system performance. Several other possible improving approaches are under way such as soft Viterbi decoding suggested in [9]. The calculation of minimum Euclidean distances for the new systems given in Sec. 3 may be too pragmatic, since there are some ambiguities around the junction areas of encoding operations. The inspection of the behaviors of trellis in these areas is in our research menu, too.

\section{References}

[1] G. Ungerboeck, "Channel Coding with Multi-Level/Phase Signals," IEEE Trans. on Inf. Theory, Jan. 1982.

[2] H. J. Viterbi, J. K. Wolf, E. Zehavi and R. Padovani, "A Pragmatic Approach to Trellis Coded Modulation," IEEE Commun. Mag., Jul. 1989.

[3] S. Sayegh, "A Class of Optimum Block Codes in Signal Space," IEEE Trans. on Comm., Oct. 1986.

[4] K. Yamaguchi and H. Imai, "Highly Reliable Multilevel Channel Coding System Using Binary Convolutional Codes," Elect. Lett., Aug. 1987. 
[5] G. J. Pottie and D. P. Taylor, "Multilevel Codes Based on Partitioning," IEEE Trans. on Inf. Theory, Jan. 1989.

[6] M.-C. Lin and S.-C. Ma, "The Performance Analysis of A Coded Modulation Scheme with Interblock Memory," 1990 Int. Symp. on Infor. Theory and Its App.

[7] —- "A Coded Modulation Scheme with Interblock Memory," to appear in IEEE Trans. on Comm.
[8] T. Woers and J. Hagenauer, "Multistage Coding and Decodng for a MPSK System," 1990 IEEE Global Telecom. Conf.

[9] - "Iterative Decoding for Multilevel Codes Using Reliability Information," 1992 IEEE Global Telecom. Conf..

[10] J. Hagenauer, "Rate-Compatible Punctured Convolutional Codes (RCPC Codes) and their Applications," IEEE Trans. on Comm., Apr. 1988.

Fig. 8. Comparison of simulated bit error rates of coded 8AMPM

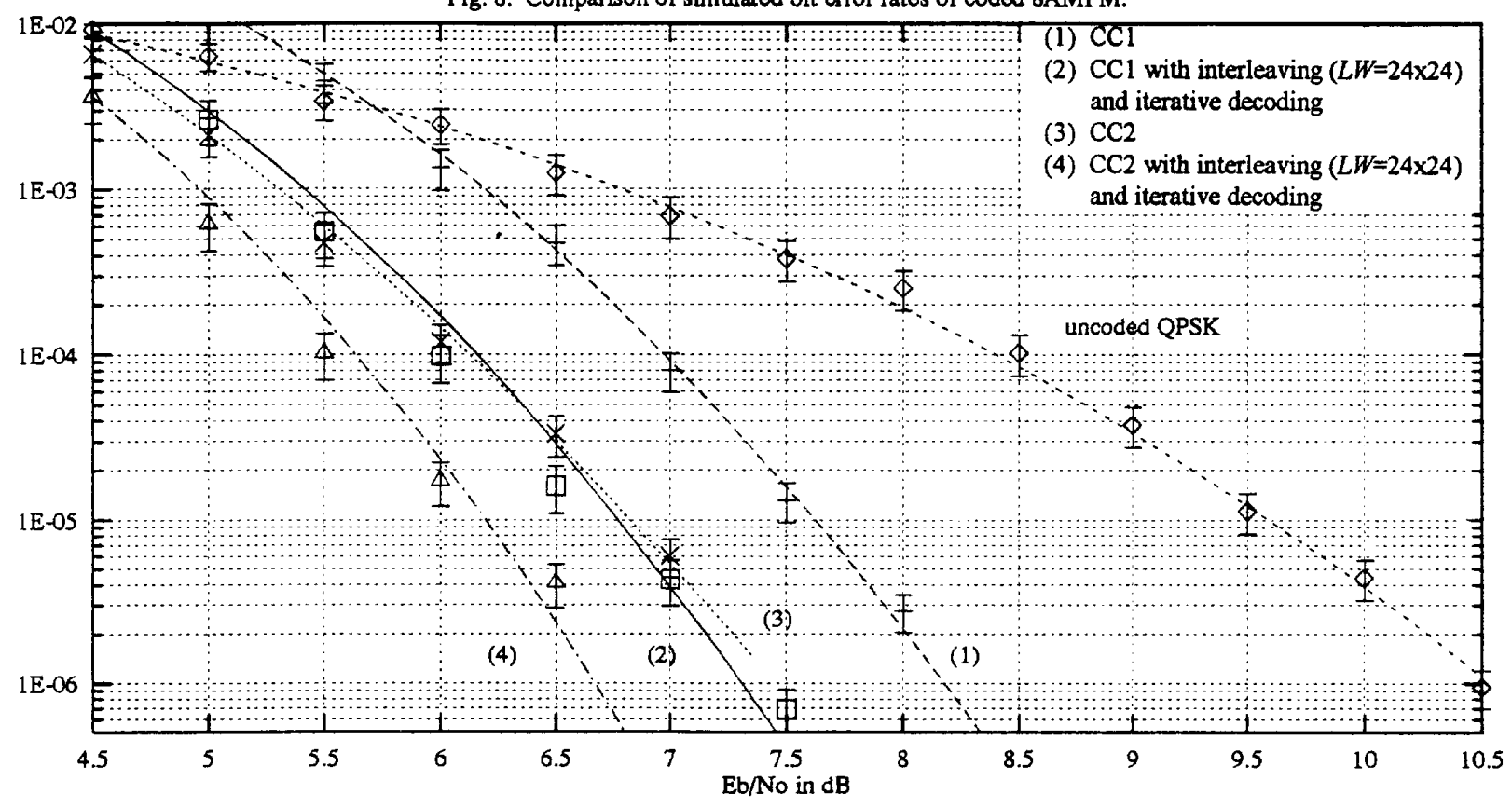

Fig. 9. Comparison of simulated bit error rates of coded 16QASK.

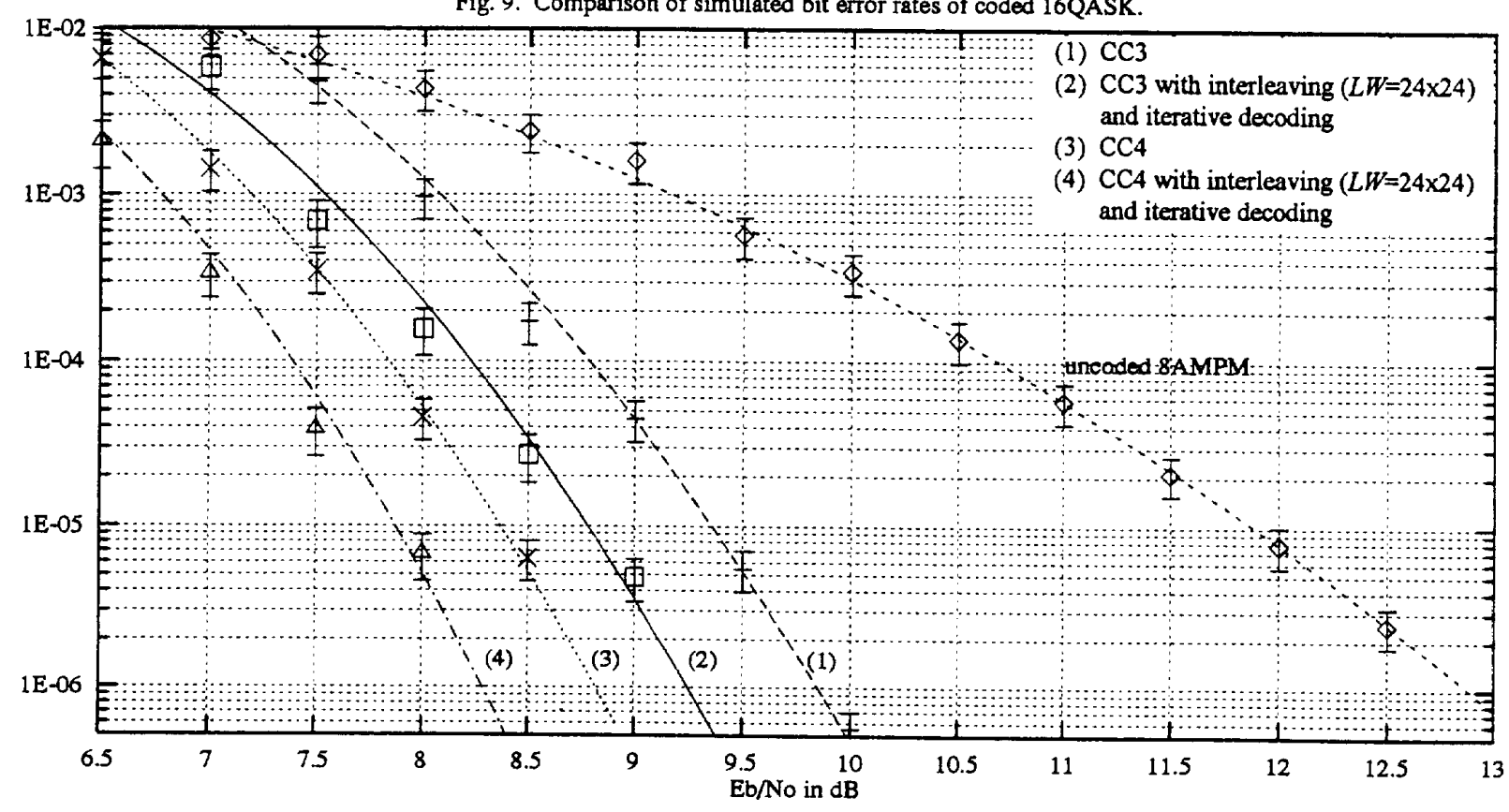

